\title{
Automated Pattern Recognition and Defect Inspection System
}

\author{
Yue Cui ${ }^{1}$, Jesse S. Jin ${ }^{1}$, Suhuai Luo ${ }^{1}$, Mira Park ${ }^{1}$, Sherlock S. L. Au ${ }^{2}$ \\ ${ }^{1}$ School of Design, Communication and IT, the University of Newcastle, Callaghan, 2308, Australia \\ ${ }^{2}$ Global Advanced Vision Pty Ltd, Room 1102, R\&D Centre, Zhonglou, Changzhou, P.R. China \\ Yue.Cui@studentmail.newcastle.edu.au
}

\begin{abstract}
Packaging appearance is extremely important in cigarette manufacturing. Typically, there are two types of cigarette packaging defects: (1) cigarette laying defects such as incorrect cigarette numbers and irregular layout; (2) tin paper handle defects such as folded paper handles. In this paper, an automated vision-based defect inspection system is designed for cigarettes packaged in tin containers. The first type of defects is inspected by counting the number of cigarettes in a tin container. First $k$-means clustering is performed to segment cigarette regions. After noise filtering, valid cigarette regions are identified by estimating individual cigarette area using linear regression. The $k$ clustering centers and area estimation function are learned off-line on training images. The second kind of defect is detected by checking the segmented paper handle region. Experimental results on 500 test images demonstrate the effectiveness of the proposed inspection system. The proposed method also contributes to the general detection and classification system such as identifying mitosis in early diagnosis of cervical cancer.
\end{abstract}

\section{Introduction}

Product inspection is a significant process in larges-scale manufacturing. Humans can inspect defects by prior knowledge and experiences. However, human vision-based inspection is hard, labor intensive, costly, inefficient due to its subjective nature. Therefore, more and more manufacturers are looking for ways using machine vision technologies to improve their productivity and reduce cost [1-3]. Hence, an efficient and reliable automated computer vision-based system is indispensible for better productivity.

Packaging appearance is extremely important in cigarette manufacturing. If there are defects in cigarette packaging, price reduces significantly. Therefore, the development of a flexible, efficient, reliable and integrated on-line vision system for cigarette packaging inspection is an essential issue in a quality control process. The automated defect inspection system is continuously conveyed in the manufacturing process and specifies whether an object is to be accepted or rejected.

Previous works in the field of automated defect inspection are mainly focused on food products [4, 5], textile $[6,7]$, tile surface $[8,9]$, wood [10], paper [11] and cigarette wrapper [12]. However, there are few publications specifically on automated defect inspection on cigarette tin packaging. Mira Park et al. [13] have proposed a cigarette packaging inspection method which can only defect cigarette laying defects. Moreover, regardless of barrel distortion of camera lens, they suppose cigarette area does not change with position, but in fact, cigarette areas are getting smaller from tin center to edge.

In this paper, we design a processing approach for cigarette packaging defect inspection system which can automatically inspect defects of cigarette packaging. The system can detect two typical types of defects: cigarette laying defects and tin container's paper handle defects. We first introduce our proposed defect inspection system framework in Section 2. The details of off-line learning procedure are presented in Section 3. In Section 4, we introduce on-line inspection procedure to detect cigarette laying defects and paper handle defects. The experimental results and discussions are then presented in Section 5. Finally, we conclude the paper in the last section.

\section{System overview}

In this paper, an automated defect inspection system is designed, which is used to defect defective cigarette packaging. The top view of each cigarette tin is captured by a camera held on the line production with appropriate illumination. The inspection system is based on image processing and pattern recognition techniques. Instead of integrating the process with too many calculations, we design the inspection system which takes less processing time and offers better accuracy. The procedure of inspection includes two typical defects: cigarette laying defects and paper handle defects. Cigarette laying defects include incorrect cigarette numbers, irregular layout and inverse placement et al. Paper handle defects include no paper handle and folded paper handle. Figure 1 displays good and defective cases respectively. The system inspects defects of (b) to (e) by counting cigarette numbers, and (f) by recognizing paper handles. 


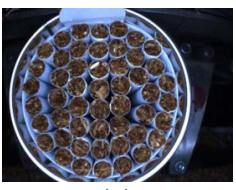

(a)

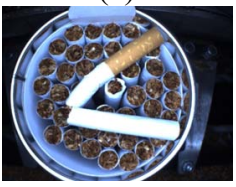

(d)

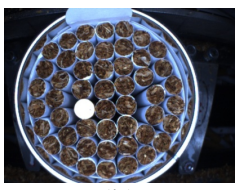

(b)

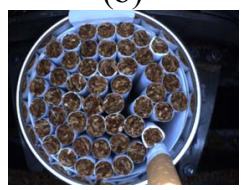

(e)

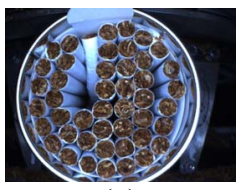

(c)

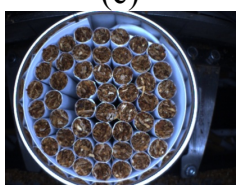

(f)
Figure 1. Good and defective cases. Image (a) is a good case with 50 regular cigarettes and a paper handle. (b) $\sim$ (f) are five defective cases. In image (b), a cigarette is inversely placed; in image (c), a cigarette is down in cigarette tin; in image (d) and (e), there are cigarettes outside the surface of tin; in image (f), the paper handle is folded.

As shown in Figure 2, the proposed inspection system framework is composed of off-line learning and on-line inspection procedure. On-line processing conveys inspection information continuously. In on-line processing, captured images pass through two types of inspections respectively. One is cigarette laying inspection the other is paper handle inspection. If any defect is detected, the system goes through defect declaration module and put the cigarette tin onto rejected product line. Paper handle inspection is composed of paper handle region segmentation and detection of paper handles. Compared with it, cigarette laying inspection is much tough, which is inspected by counting the number of cigarettes in our approach. First k-means clustering is performed to segment cigarette regions. Then noise filtering is employed to fill holes and separate connected cigarette regions, finally valid cigarette regions are identified by estimating individual cigarette area using linear regression. The $\mathrm{k}$ clustering centers and area estimation function are learned off-line on training images. In off-line learning, k-means clustering in HSI color space obtains $\mathrm{k}$ clustering centers for on-line cigarette regions segmentation; linear regression finds a best fit area estimation function $S_{c i g}=f(d)$ in order to predict the area of individual cigarettes at different locations.

\section{Off-line learning}

Off-line learning is performed on training images with good cases before on-line inspection procedure. It provides inputs for cigarette laying defects inspection.

First of all, the background of images should be removed in order to focus on the region of interest. We observe the circle of cigarette tin is highlight because of metal materials. We can make use of this character to cut away background of the captured images, eliminate mirror effect around tin, and obtain the radius and center of the tin.

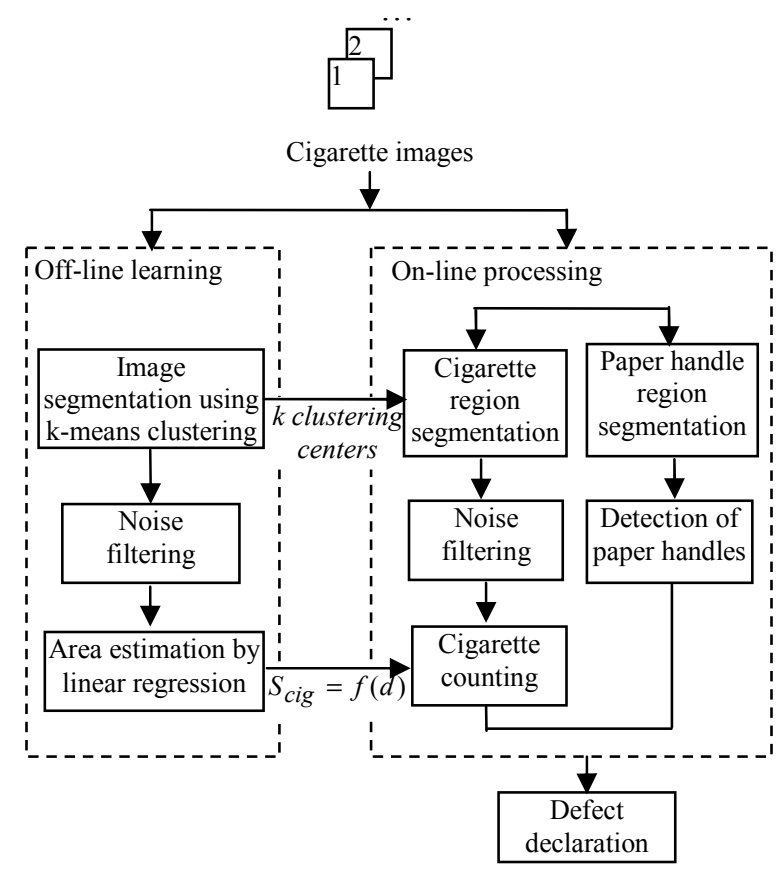

Figure 2. Framework of defect inspection system

\subsection{K-means clustering}

Original data from a color camera are digitized RGB (Red, Green and Blue) images. It is more common to describe a color by RGB components on computers, but as humans, we cannot easily extract each color from RGB representation. Therefore, in machine vision, the most used color representation is the hue, saturation and intensity (HSI) color model which is based on human color perception. HSI color space is more precise in describing color, which consists of hue angle, color saturation and intensity. Comparison results in RGB and HSI color space $\mathrm{k}$-means clustering and segmentation are shown in Figure 3. Original image is Figure 1(a). We employ k-means clustering algorithm in RGB and HSI color space and segment cigarette regions respectively. We observe in RGB color space, brown color of cigarettes and dark colors around cigarettes cannot be separated after k-means clustering, while they can be obviously separated in HSI color space. The different results demonstrate HSI color space is much better for segmentation in this case. Hence, we convert RGB color space into HSI color space.

After obtaining the hue, saturation and intensity of each image, we employ k-means clustering algorithm to segment cigarette regions. As we do not know which $\mathrm{k}$ value is best beforehand, we set $\mathrm{k}$ from 3 to 10 in testing images. Best results are achieved when $\mathrm{k}$ is set to 5 , as seen in figure 3 . We average centers of each training image as five clustering centers for classification for on-line images. We 
colorize two classes of pixels white which are region of interest, three left classes of pixels black which are background. Then the images are converted into binary images for simplification.
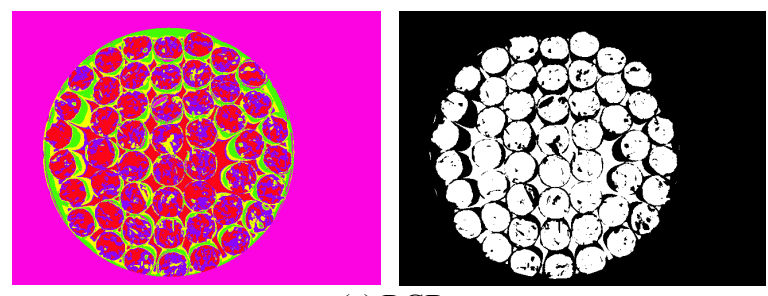

(a) RGB
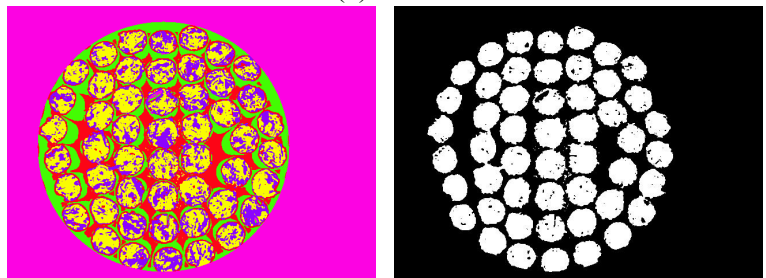

(b) HSI

Figure 3. Image segmentation results using k-means clustering in RGB and HSI color space. We observe in RGB color space, brown color of cigarettes and dark colors around cigarettes cannot be separated after k-means clustering, while they can be obviously separated in HSI color space. The different results demonstrate HSI color space is much better for segmentation in this case.

\subsection{Noise filtering}

After the segmentation stage, we observe in Figure 3(b) that the segmentation suffers from inaccuracy because there are many black holes belonging to the foreground. Such noises interfere with identification of cigarettes, therefore noise filtering must be achieved. Many connected cigarettes should also be separated from each other. In this stage, we appeal to seed filling algorithm [14] and morphological operation for the further processing.

Seed filling algorithm is employed on the right image in Figure 3(b), then erosion operations are used twice. Results are seen in Figure 4(a) and (b). We observe black holes presented in the segmented binary image are filled after seed filling algorithm, connected regions are mostly disconnected by erosion operation.

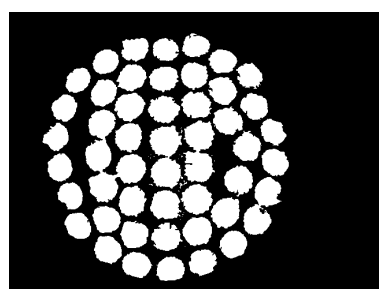

(a)

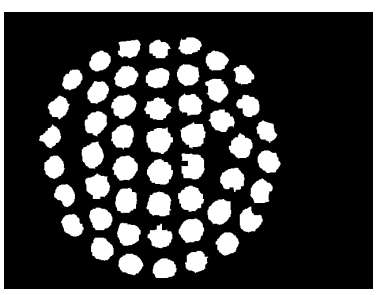

(b)
Figure 4. Results of seed filling (a) and erosion operations (b). We observe black holes on cigarette regions are filled after seed filling algorithm, connected regions are mostly separated by erosion operation.

\subsection{Regression analysis}

After noise filtering, most of cigarettes are disconnected but some are still physically connected. Figure 5 illustrates results of noise filtering, (a) is the original image, (b) shows two connected cigarette regions in red after noise filtering.

In order to count cigarette numbers, we need to identify valid cigarette regions. Due to barrel distortion of camera lens, the areas of cigarettes are getting smaller from the center to edge. So we need to find the area of an object in relation to the distance to the center in order to count the number of cigarettes correctly. We define the area estimation problem as a regression problem.

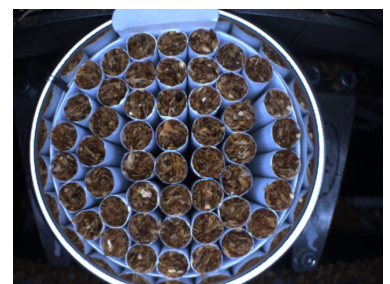

(a)

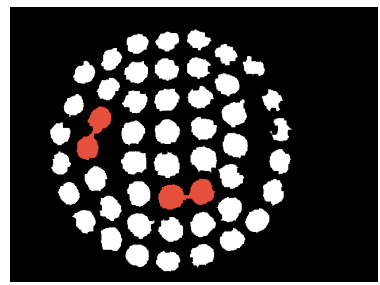

(b)
Figure 5. Results of noise filtering. (a) original image; (b) two connected cigarette regions in red after noise filtering. We observe most of cigarette regions are separated, but some are still physically connected.

Given a sequence of observations

$$
\left(d_{1}, S_{1}\right), \cdots,\left(d_{n}, S_{n}\right)
$$

Where $d_{i}$ is the distance from tin center to geometric center of an independent object region, $S_{i}$ is the area of an independent object region, $d_{i}, S_{i} \in R$. Equation (1) specifically describes the regression model

$$
S_{\text {cig }}=f(d)
$$

Where $f(\cdot)$ is the estimated regression function. A typical choice of the regression function $f$, is the linear model, namely

$$
S_{\text {cig }}=\beta_{0}+\beta_{1} x
$$

The model parameters are optimized by minimizing the difference between the actual area of objects and the estimated area. Linear model is enough in such a scenario because the size of image is not large. We select disconnected (independent) cigarette objects in training images as samples, obtain areas by seed filling algorithm and distance from disconnected region geometric center to tin center. Figure 6 displays the best fit line of given distance and area of objects. We observe that the regression 
function can estimate the global object area trends. Cigarettes near the center of tin have bigger area about 1400 pixels, cigarettes near edge of tin have smaller area about 1000 pixels.

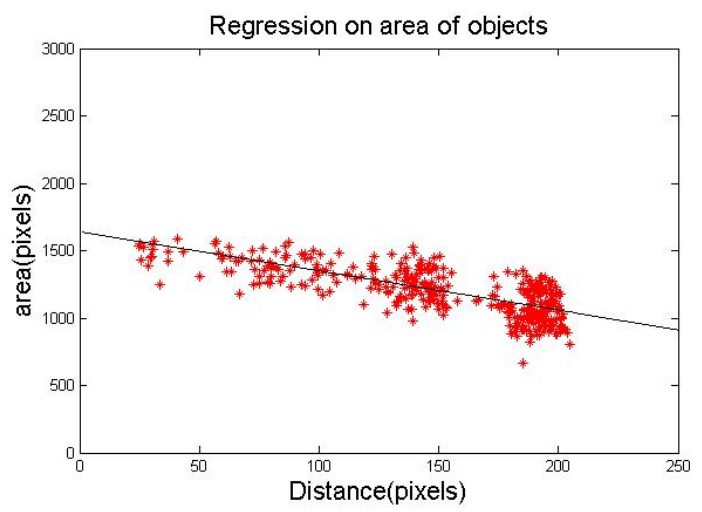

Figure 6. Linear regression on distance $\left(d_{i}\right)$ and area $\left(S_{i}\right)$ of objects

\section{On-line inspection}

On-line inspection procedure inspects cigarette laying defects and paper handle defects respectively. The first type of defects is more common and tough which is inspected by counting the number of cigarettes in our approach, the other type of defects is inspected by identification of paper handles.

\subsection{Cigarette counting}

As explained in off-line section, all on-line captured images should perform pre-processing, namely color space conversion from RGB to HSI. In HSI color space, five clustering centers which obtained in off-line learning are used to classify pixels. Images are converted into binary images by filling pixels of cigarette regions white and others black. Then noise filtering is employed to fill the possible holes presented in the segmented binary image and separate physically connected regions. Given the regression function in off-line learning, we can estimate cigarette area $S_{c i g}$ by the distance of geometric center to the center of the tin. We can obtain each connected white region area $S_{\text {region }}$ by seed filling algorithm, and distance of geometric center of white region to the center of the tin, so we divide the area of the region $S_{\text {region }}$ with estimated

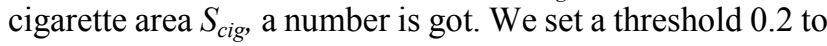
identify objects. If the number is within 0.2 of an integer and the integer is a positive number, i.e.

$$
\left|S_{\text {region }} / S_{\text {cig }}-\left[S_{\text {region }} / S_{\text {cig }}+0.5\right]\right|<0.2
$$

where $\left[S_{\text {region }} / S_{\text {cig }}+0.5\right]$ returns a nearest integer that is smaller than the value inside the square brackets, we regard it as a valid cigarette region with the integer cigarettes.
Otherwise, we label it as an invalid cigarette region.

After finding the number of cigarettes, we can use the number as $\mathrm{k}$ to pass the region to $\mathrm{k}$-means clustering.

After obtaining $\mathrm{k}$ centers, we draw a circle around each center using $S_{\text {region }}=\pi \rho^{2}$ to obtain $\rho$, where $S_{\text {region }}$ is the area of the region. We then check the ratio of white and black area inside the circle to decide the correctness of the cigarettes.

\subsection{Inspection of paper handles}

The right image in Figure 1(f) shows another defective case, i.e. the paper handle is folded. Paper handles should be white and near the tin edge. So we define paper handle region a ring shape around tin edge. We use the center and radius of cigarette tin which obtained in cigarette counting stage to cut out paper handle regions, and convert it into a grayscale image (Figure 7(a)). A binary image is generated by converting pixels with grayscale values ranging from 170 to 230 into values of 1 (white areas in Figure 7(b)), other pixels into values of 0 (black areas), because pixels near tin edge are mostly larger than 230 due to material character. In this way paper handle area is converted into a white block. Figure 7 illustrates the effect of grayscale image and binary image. We detect existence of paper handle by calculating the area of the white region. If the area is larger than 3500 pixels, the system passes it as a good case in paper handle.

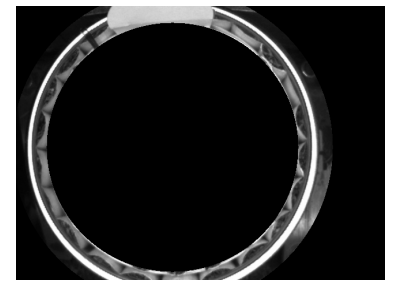

(a)

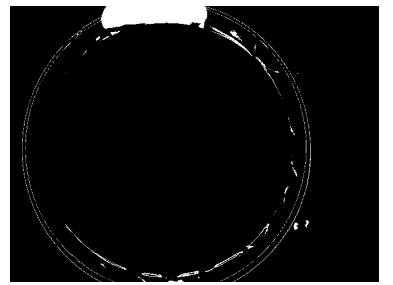

(b)
Figure 7. Detection of paper handle. (a) grayscale image with paper handle region; (b) binary image with paper handle region

The system can inspect all defective cases in Figure 1. Figure 8 shows two defective results.
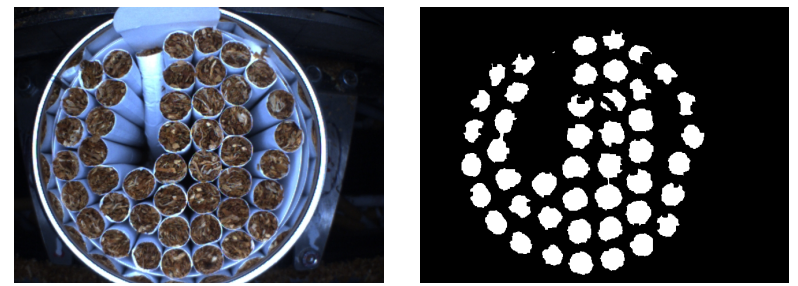

(a) 

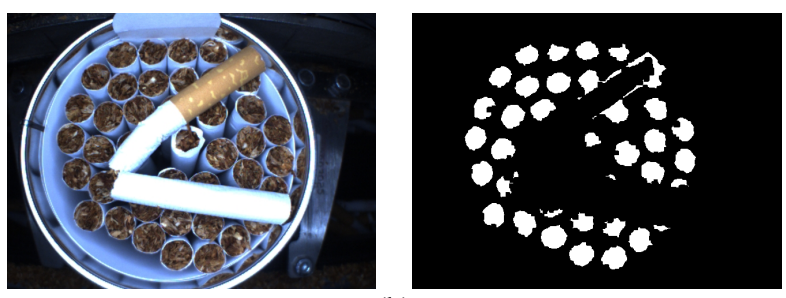

(b)

Figure 8. Two defective results (a) and (b). Original defective images are on the left, processing results are on the right.

\section{Experimental results}

Our data set has 500 cigarette packaging images including 400 good images and 100 defective images, among which there are 11 images with paper handle defects. We evaluate our method using sensitivity and specificity by Equation (4).

$$
\begin{aligned}
& \text { Sensitivity }=\frac{\text { TruePositive }}{\text { TruePositive }+ \text { FalseNegative }} \\
& \text { Specificity }=\frac{\text { TrueNegative }}{\text { TrueNegative }+ \text { FalsePositive }}
\end{aligned}
$$

where TruePositive means the number of defective cases correctly detected as defective; FalseNegative means the number of defective cases wrongly detected as good; FalsePositive means the number of good cases wrongly detected as defective; TrueNegative means the number of good cases correctly detected as good.

Our method can detect all of defective cases efficiently (sensitivity $=100 \%$ ). The specificity is $96.5 \%$. Compared with our improved method, previous method proposed by Mira Park et al. [13] only detect cigarette laying defects using Lab color space without regression analysis, which supposes cigarette area does not change with distance. Mira's test is only on 18 images including 8 good cases and 10 defective cases. We compare our results with Mira's method on a larger data set as mentioned above, and paper handle defect images are excluded. Results are shown in Table 1, our method brings $3.2 \%$ improvement in specificity in cigarette laying defects inspection.

Results demonstrate that the proposed method is capable of detecting most of defects for cigarette packaging. The inspection system offers high sensitivity and specificity. It can be used in cigarette packaging industry effectively.

Table 1. Comparisons with Mira's method of cigarette laying defects inspection

\begin{tabular}{|c|c|c|}
\hline & Sensitivity & Specificity \\
\hline Mira's method & $100 \%$ & $93.3 \%$ \\
\hline Our method & $100 \%$ & $96.5 \%$ \\
\hline
\end{tabular}

\section{Conclusions}

In this paper, an automated cigarette defect inspection system is designed for cigarette packaging. The proposed system is composed of off-line learning module and on-line inspection module. Off-line learning consists of k-means clustering and linear regression. K-means clustering in HSI color space obtains $\mathrm{k}$ cluster centers for on-line cigarette region segmentation. Linear regression on area of objects is introduced to estimate area of an independent object for identifying valid cigarette regions. On-line procedure inspects two types of defects respectively: cigarette laying defects and paper handle defects. Cigarette laying defects such as incorrect cigarette numbers and irregular layout are inspected by counting cigarette numbers in a cigarette tin. First we use k-means clustering to segment cigarette regions. Then noise filtering is employed to fill holes and separate connected regions. At last valid cigarette regions are identified by estimating individual cigarette area on linear regression function. Paper handle defects such as folded paper handles are inspected by checking segmented paper handle region. The proposed method is tested on 500 images. The experimental results demonstrate the system can be used in cigarette packaging industries effectively with high feasibility.

The research is also the part of our research on mitosis detection and classification in early diagnosis of cervical cancer. The proposed method significantly contributes to the methodology development of new algorithms.

\section{Acknowledgement}

This project is partially supported by CSC-Newcastle Scholarship and ARC LP0669645 grant.

\section{References}

[1] U.S. Khan, J. Iqbal, and M.A. Khan, "Automatic inspection system using machine vision", Proceedings of the $34^{\text {th }}$ applied Imagery and Pattern Recognition Workshop (AIPR05), IEEE Computer Society, 210-217, 2005.

[2] B.G. Batchelor, and P.F. Whelan, Intelligent Vision Systems for Industry, Springer-Verlag Inc, New York, 1997.

[3] T. Brosnan, D.W. Sun, "Improving quality inspection of food products by computer vision - a review", Journal of Food Engineering, 61: 3-16, 2004.

[4] J.C.H. Yeh, L.G.C. Hamey, T. Westcott and S.K.Y. Sung, "Colour bake inspection system using hybrid artificial neural networks", IEEE International Conference on Neural Networks, Perth, WA, Australia, 37-42, 1995.

[5] K.L.Yam, and S.E. Papadakis, "A simple digital imaging method for measuring and analyzing color of food surfaces", Journal of Food Engineering, 61: 137-142, 2004.

[6] A. Kumar, G. K. H. Pang, "Defect detection in textured materials using gabor filters", IEEE Transactions on Industry Applications, 38(2):425-440, 2002.

[7] S. Kim, H. Bae, S.P. Cheon and K.B. Kim, "On-line fabric-defects detection based on wavelet analysis", ICCSA, Springer-Verlag, Berlin Heidelberg, 1075-1084, 2005. 
[8] C.E. Costa, M. Petrou, "Automatic registration of ceramic tiles for the purpose of fault detection", Machine Vision and Applications, Springer-Verlag, 225-230, 2000.

[9] H. Elbehiery, A. Hefnawy, and M. Elewa, "Surface defects detection for ceramic tiles using image processing and morphological techniques", Proceedings of word academy of science, engineering and technology, 158-162, 2005.

[10] E. Young, "Use of linescan camera and a DSP processing system for high-speed wood inspection", Machine Vision Applications, Architectures, and Systems Integration IV, vol.2597 of Proceedings of SPIE, Philadelphia, USA, 259-264, 1995.

[11] J.W. Roberts, S.D. Rose and G.A. Jullian, "PC based real-time defect imaging system for high speed web inspection", Machine Vision Applications in Industrial Inspection, vol.1907 of Proceedings of SPIE, San Jose, USA, 164-176, 1993.

[12] Z. Zhu, Y. Guo, "Print quality detection of cigarette wrapper based on image processing and BP network", Proceedings of the $7^{\text {th }}$ World Congress on International Control and Automation, Chongqing, China, 2253-2258, 2008.

[13] Mira Park, Jesse S. Jin, Sherlock L. Au, Suhuai Luo, "Pattern recognition from segmented images in automated inspection systems", International Symposium on Ubiquitous Multimedia Computing, New Zealand, 87-92, 2008.

[14] R.C. Gonzalez and R.E. Woods, Digital Image Processing, Prentice Hall, 2008. 